\title{
BMJ Open ENACT (ENvironmental enrichment for infants; parenting with Acceptance and Commitment Therapy): a randomised controlled trial of an innovative intervention for infants at risk of autism spectrum disorder
}

Koa Whittingham (D) , ${ }^{1}$ Andrea McGlade, ${ }^{1}$ Kavindri Kulasinghe, ${ }^{1}$ Amy E Mitchell, ${ }^{2}$ Honey Heussler, ${ }^{3,4}$ Roslyn N Boyd ${ }^{1}$

To cite: Whittingham K, McGlade A, Kulasinghe $\mathrm{K}$, et al. ENACT (ENvironmental enrichment for infants; parenting with Acceptance and Commitment Therapy): a randomised controlled trial of an innovative intervention for infants at risk of autism spectrum disorder. BMJ Open 2020;10:e034315. doi:10.1136/ bmjopen-2019-034315

- Prepublication history and additional material for this paper are available online. To view these files, please visit the journal online (http://dx.doi. org/10.1136/bmjopen-2019034315).

Received 14 September 2019

Revised 04 May 2020

Accepted 08 June 2020

Check for updates

(C) Author(s) (or their employer(s)) 2020. Re-use permitted under CC BY-NC. No commercial re-use. See rights and permissions. Published by BMJ.

For numbered affiliations see end of article.

Correspondence to Dr Koa Whittingham; koawhittingham@uq.edu.au

\section{ABSTRACT}

Introduction Autism spectrum disorder (ASD) is a heterogeneous neurodevelopmental condition with impacts on behaviour, cognition, communication, social interaction and family mental health. This paper reports the protocol of a randomised controlled trial (RCT) of a very early intervention, ENACT (ENvironmental enrichment for infants; parenting with Acceptance and Commitment Therapy), for families of infants at risk of ASD.

Methods and analysis We aim to recruit 66 mothers of infants at risk of ASD (ie, infants with a sibling or parent diagnosed with ASD) to this RCT. Families will be randomly assigned to care-as-usual or ENACT. ENACT is a very early intervention, leveraging parent-child interactions to improve early social reciprocity, while supporting parental mental health and the parent-child relationship through Acceptance and Commitment Therapy. Intervention content is delivered online (approximately 8 hours) and supported by more than 7 consultations with a clinician. Parents will perform the social reciprocity intervention with their child (30 min per day). Assessments at four time points (baseline, 3 months, 6 months, and 12 months corrected age) will assess parentinfant interaction, parental mental health, infant development and early ASD markers. Analysis will be by intention to treat using general linear models for RCTs.

Ethics and dissemination This protocol has been approved by the Children's Health Queensland Hospital and Health Service Human Research Ethics Committee (HREC/19/ QCHQ/50131) and the University of Queensland Human Research Ethics Committee (2019000558). If efficacy is demonstrated, the intervention has the potential for wide and accessible dissemination.

Trial registration number Australian New Zealand Clinical Trials Registry (ACTRN12618002046280).

\section{INTRODUCTION}

Autism spectrum disorder and the broader autism phenotype

Autism spectrum disorder (ASD) is a heterogeneous neurodevelopmental condition defined by difficulties in social
Strengths and limitations of this study

- First randomised controlled trial to test a very early intervention for infants at risk of autism spectrum disorder implemented within the first 6 months of life.

- ENACT (ENvironmental enrichment for infants; parenting with Acceptance and Commitment Therapy) combines parent-mediated very early intervention with parental mental health support.

- Assessment includes neurodevelopmental assessments, as well as observations of parent-child interaction.

- ENACT is designed to be delivered by health professionals with relevant clinical experience in working with parents and in Acceptance and Commitment Therapy, and could, if effective, be widely disseminated at little cost.

communication and interaction, and repetitive, restricted interests and activities. ${ }^{12}$ It evolves from a complex interaction between genes and environment, ${ }^{34}$ and has substantial impact on affected individuals, with $65 \%$ having a profound or severe activity limitation, needing help or supervision with communication, self-care and/or mobility. ${ }^{5}$ Prevalence rates are $0.7 \%$ and $1.7 \%-2.5 \%$ in Australia and the United States, respectively. ${ }^{5-7}$ Diagnosis rests on developmental assessment and behavioural observations, with most children 2 years or older at diagnosis. ${ }^{8}$

Infant siblings of children with ASD are at an increased risk of ASD themselves, with prevalence estimates of $18 \%-20 \%$ from baby sibling studies. ${ }^{9}{ }^{10} \mathrm{~A}$ further $25 \%$ show elevated scores on the Autism Diagnostic Observation Schedule, developmental delays 
and lower adaptive functioning. ${ }^{11-13}$ Prospective sibling studies have identified a range of non-specific markers in infants at a high risk of ASD, including motor delays, poor visual reception, language delays, regulatory difficulties and changes in eye gaze at 6-12 months that precede the appearance of autism-specific features in the second year of life. ${ }^{14-18}$ The diversity of early markers precludes a single developmental pathway to ASD and has been called 'the first year puzzle'. ${ }^{19-23}$ Non-specific developmental markers may interact leading to increasingly abnormal trajectories of infant development. Visual, motor and regulatory difficulties may impact on emerging attention and emotional regulation. ${ }^{192-26}$ Differences in visual, motor and regulatory abilities at 6 months of age correspond in timing with changes in whole-brain functional connectivity on MRI studies. ${ }^{27}{ }^{28}$ At 6 months of age, functional connectivity on diffusion tensor imaging MRI correctly predicted 9 of 11 infants that went on to be diagnosed with ASD at 24 months of age. ${ }^{29}$ These findings support the conjecture that the developmental cascade leading to ASD begins early, within the first 6 months of life. $^{28}$

To date, nine randomised controlled trials (RCTs) have tested parent-mediated early interventions with infants at risk of ASD implemented in the first 24 months, prior to confirmed ASD diagnosis. ${ }^{30-36}$ Only one of these, an RCT of Intervention within the British Autism Study of Infant Siblings- Video Interaction to Promote Positive Parenting Programme (iBASIS-VIPP), conducted with 54 infants at high familial risk of ASD recruited at 7-10 months of age has demonstrated sustained reduction of ASD-related symptoms, but no change in the diagnostic outcome at 3 years. ${ }^{30}{ }^{34}$ 37-39 iBASIS-VIPP begins after the infant is 6 months of age and focuses on changing parent behaviour. To date, no RCT has commenced with at-risk infants before the infants reach 6 months of age, before earliest ASD markers and commencement of the cascade.

\section{ASD in the family context}

Poor maternal mental health contributes to poorer longterm outcomes for infants, including those at risk of ASD. ${ }^{40}$ Parents of children with ASD are at increased risk for depression and anxiety, ${ }^{42}{ }^{43}$ both due to parenting challenges and pre-existing histories. ${ }^{44-46}$ Parents of infants with ASD are more likely to have an ASD or the broader autism phenotype (BAP). ${ }^{47-50}$ The BAP may include rigidity, aloofness, social and communication difficulties ${ }^{50}$ with increased risk of depressive symptomatology, maladaptive coping and decreased social support. ${ }^{50}$

Mental health difficulties can contribute to reductions in responsive parenting. ${ }^{51}$ Responsive parentingchild-directed, contingent, prompt and appropriate to the child's needs-is associated with better child outcomes. ${ }^{52}{ }^{53}$ Responsiveness is a dose-control system for environmental enrichment, enabling the child to obtain the necessary 'experience expectant development'. ${ }^{54} 55$ If a child is difficult to read, sends atypical or unclear signals, as in ASD, it is more challenging for parents to cultivate responsive patterns of interaction. ${ }^{56}$

By 6 months, infants at risk of ASD may be showing an atypical style of interaction, with difficulty engaging in eye contact and joint attention. ${ }^{15} 26535758$ Parent behaviour may shift towards intrusive parenting and high-intensity approach behaviours in an attempt to foster engagement and overcome the emerging social limitations of ASD. ${ }^{53}$ The shift to directive parenting may impact further on the infant's social development. ${ }^{5360}$ Importantly, commencing parent-focused intervention prior to 6 months, before the shift towards directive parenting, has not been tested.

\section{Aim}

To test the efficacy of ENACT (ENvironmental enrichment for infants; parenting with Acceptance and Commitment Therapy) for families of infants at risk of ASD via an RCT comparing ENACT to care-as-usual (CAU). ENACT is a newly developed, very early intervention that targets infants' social reciprocity through supported parentinfant interactions, while simultaneously supporting parental mental health and the parent-child relationship. ENACT commences prenatally.

\section{Hypotheses}

We predict that families allocated to ENACT will show better outcomes compared with families allocated to CAU in terms of having the following.

\section{Primary outcomes}

- H1: lower scores for ASD symptomatology as assessed on (a) the Autism Observation Schedule in Infants (AOSI) ${ }^{61} 62$ at 12 months and (b) the greater ease of disengagement and greater reduction in the gap effect (reaction time at overlap minus reaction time at gap) on the gap-overlap task at 12 months in comparison to 6 months. ${ }^{6364}$

\section{Secondary outcomes}

- H2: better scores on measures of parents' mental health at 3 months, 6 months and 12 months as assessed on the Depression, Anxiety Stress Scale-21 items (DASS-21) and the Acceptance and Action Questionnaire-II (AAQ-II). ${ }^{65} 66$

- H3: improved parent-infant interaction, with greater emotional availability and parental sensitivity, less parental intrusiveness and greater child responsiveness, as assessed on the Emotional Availability Scales (EAS): self-report at 3 months, 6 months and 12 months and observed ${ }^{67}$ at 6 months.

- H4: higher scores on measures of (a) infants' cognitive development, assessed using the Mullen Scales of Early Learning (MSEL) Early Learning Composite (ELC) at 6 months and 12 months (a composite of the subdomains of visual reception (VR), fine motor (FM), receptive language (RL) and expressive language (EL)), ${ }^{68}$ and (b) infants' adaptive skills, 
assessed using the Vineland Adaptive Behavior Scales, Third Edition (VABS-3) ${ }^{69}$ at 12 months.

- H5: higher scores on measures of infants' (a) motor development at 6 months and 12 months assessed using the Infant Neurological Examination (HINE) ${ }^{70} 71$ and (b) fine and gross motor abilities (assessed using the MSEL). ${ }^{72}$

- H6: higher scores on measures of infants' visual perceptual skills at 6 months and 12 months, assessed on the VR scores on the MSEL, ${ }^{24}$ and on symbolic cluster on the Communication and Symbolic Behavior Scales-Developmental Profile (CSBS-DP) ${ }^{73}$ and with reduced times on the gap-overlap task.

- H7: higher scores on measures of infant language development at 6 months and 12 months, assessed by the RL and EL domains on the MSEL ${ }^{74}$ and CSBS-DP. ${ }^{75}$

- H8: better scores on parent-report measures of infant regulation-specifically, (a) lower scores for internalising and externalising behaviour, assessed on the Infant-Toddler Social and Emotional Assessment (ITSEA) at 12 months $^{76}$; (b) lower scores on the dysregulation scales of the ITSEA at 12 months; (c) better sleep on the Brief Infant Sleep Questionnaire (BISQ) at 3 months and 6 months ${ }^{77}$ and (d) less cry behaviours on the Crying Pattern Questionnaire $(\mathrm{CPQ})$ at 3 months and 6 months of age. ${ }^{78}$

\section{METHODS AND ANALYSIS}

\section{Design}

The study is an RCT following the Consolidated Standards of Reporting Trials (CONSORT) guidelines. After enrolment, and baseline assessments, mothers of infants at risk of ASD will be randomly allocated to intervention (ENACT) or CAU. Comparison to CAU is appropriate as ENACT is a newly developed intervention and this is the first trial. The CONSORT flow chart is depicted in figure 1 .

\section{Recruitment}

Families will be recruited via advertisements distributed through Queensland ASD family support groups, schools and clinics (eg, Autism Queensland, AEIOU Foundation for Children with Autism, Asperger Services Australia and Minds and Hearts) and Queensland Health Antenatal and Child Development Clinics. Families will be recruited during pregnancy and up to the infant reaching 7 weeks corrected age (CA) then followed over the first 12 months.

\section{Inclusion criteria}

Participants must meet the following inclusion criteria: (1) the infant must have one or more biological siblings or a biological parent (mother or father) diagnosed with ASD; (2) the mother must agree to the assessment requirements; (3) the mother must have reliable internet access and (4) the mother must have sufficient English to complete assessments.

\section{Exclusion criteria}

Any infant with known neurological or chromosomal disorder at the point of recruitment.

\section{Sample size}

The target number of participants is 66 (ENACT, $n=33$; CAU, $n=33$ ), which will provide power of $80 \%$ (two-tailed, $\alpha=0.05$ ) to detect a difference between groups of $0.75 \mathrm{SD}$ on the AOSI. In a previous study with a similar sample, the observed SD was $4 ;{ }^{30}$ consequently, we should be able to observe a difference of $\geq 3$ units in this study.

\section{Blinding}

Participants and intervention delivery facilitators cannot be blinded to group allocation. Assessors conducting the AOSI, gap-overlap task, MSEL and HINE assessments at 12 months CA will be blinded to group allocation, as will coders scoring the video-recorded/audio-recorded EAS observations, General Movements Assessment (GMA) and gap-overlap task.

\section{CAU interventions for infants at risk of ASD}

Participants allocated to CAU will receive usual postnatal care. As developmental and autism-related concerns generally present after 12 months of age, it is expected that any targeted interventions provided in the community by usual care providers will fall outside the timeframe of the study.

\section{The ENACT intervention}

ENACT is a very early intervention targeting infant social reciprocity through supported parent-child interactions while simultaneously supporting parental mental health and the parent-child relationship using Acceptance and Commitment Therapy (ACT). Core to ENACT is the social reciprocity intervention which teaches mothers to initiate and build sensitivity chains with their babies, with the goal that sensitivity chains become longer, increasingly complex and increasingly symbolic over time, and that the early social development of the infant is optimally supported. They should be mutually enjoyable, responsive and non-intrusive.

The three simple steps to building a sensitivity chain are for the mother to (1) stimulate an initial enjoyable interaction, (2) wait for the infant to signal their intent to continue and (3) respond to the infant's signal, hence 'closing the loop' and building a link in the sensitivity chain. This will include a focus on: initially, cultivating sensitivity chains through sensorimotor activities, using positive affect and predictable surprise to support the infant's involvement, maintaining reciprocal interactions with infants with atypical responsiveness and avoiding parental intrusiveness with atypically responsive infants. This intervention is specifically targeting the earliest documented abnormalities in social behaviour in infants at risk of ASD. ${ }^{79}$ This aspect of the ENACT intervention was developed specifically for this trial by AMG, with input from KW. 


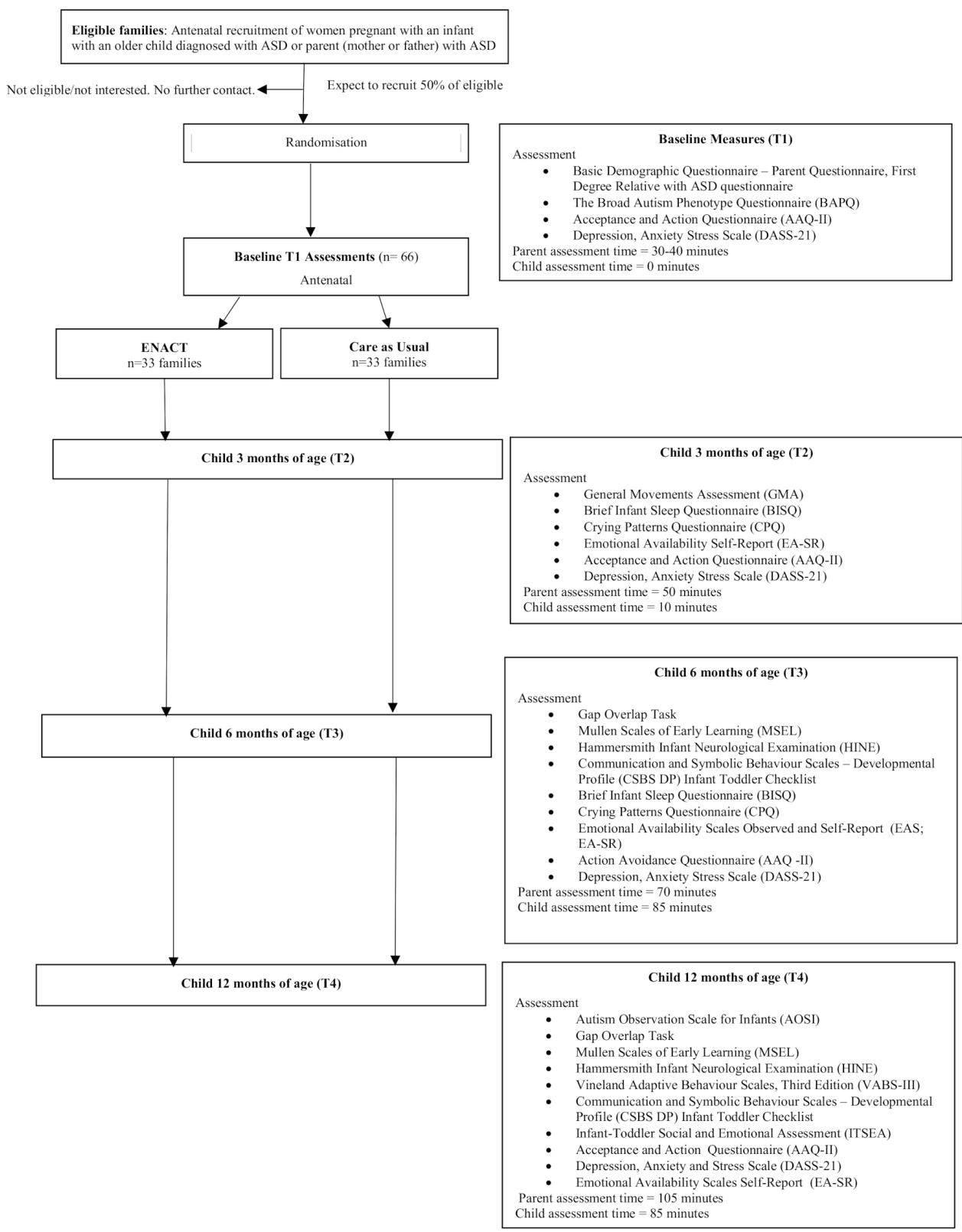

Figure 1 Consort flow chart of the ENACT (ENvironmental enrichment for infants; parenting with Acceptance and Commitment Therapy) study.

ENACT also incorporates parental mental health support grounded in ACT, including values, mindfulness, experiential acceptance and cognitive defusion (distancing from thoughts). The ACT component within ENACT contains material previously trialled as the online intervention entitled Parenting Acceptance and Commitment Therapy or PACT. ${ }^{51}$ The development of PACT was itself grounded in RCTs demonstrating the efficacy of a group ACT intervention for parents of children with neurodevelopmental disabilities. ${ }^{80-83}$ ENACT also contains a small psychoeducation component on common early parenting challenges of sleep, crying and feeding, developed by KW and grounded in the existing literature and her previous work. ${ }^{84-87}$ This focuses on understanding the biological regulation of sleep via the circadian clock and the sleep-wake homeostat, understanding the developmental pattern of infant crying, including the crying peak, and planning ahead on where to seek help for feeding challenges.

ENACT is delivered to mothers (1) via an online course (approximately 8-hour duration) using the edX platform (www.edx.org/) and (2) through telehealth (videoconferencing via Zoom) consultations with a trained clinician. The edX course includes: videos and text explaining core concepts, interactive exercises, multiple choice questions and videos of real parent-and-baby interactions. The social reciprocity very early intervention is delivered to the infant through the mother and other caregivers. Intervention delivery to mothers will commence prenatally, and mothers will receive fortnightly sessions with the clinician to support consolidation of learning. Mothers will be encouraged to work through the edX course at 
Table 1 ENACT intervention components

\begin{tabular}{|c|c|c|}
\hline Component & Timing & Content \\
\hline \multicolumn{3}{|l|}{ ENACT edX course } \\
\hline $\begin{array}{l}\text { Module 1: very early intervention } \\
\text { approach }\end{array}$ & $\begin{array}{l}\text { Begins antenatally with completion } \\
\text { before child is } 8 \text { weeks }\end{array}$ & $\begin{array}{l}\text { Outlines the very early intervention approach or sensitivity } \\
\text { chains and includes multiple videos of parents and babies. } \\
\text { Includes advice on early parenting challenges. }\end{array}$ \\
\hline Module 2: living a meaningful life & $\begin{array}{l}\text { Begins antenatally with completion } \\
\text { before child is } 8 \text { weeks }\end{array}$ & $\begin{array}{l}\text { Grounded in ACT, focuses on values and living a rewarding } \\
\text { life. }\end{array}$ \\
\hline Module 4: relating to others & $\begin{array}{l}\text { Begins antenatally with completion } \\
\text { before child is } 8 \text { weeks }\end{array}$ & $\begin{array}{l}\text { Grounded in ACT, focuses on acceptance, compassion and } \\
\text { flexible parenting. }\end{array}$ \\
\hline Module 5: extending early intervention & $\begin{array}{l}\text { Begins antenatally with completion } \\
\text { before child is } 8 \text { weeks }\end{array}$ & $\begin{array}{l}\text { Extends sensitivity chain practice for older babies and } \\
\text { provides advice for parents of babies experiencing } \\
\text { challenges. }\end{array}$ \\
\hline Developmental consultations & $\begin{array}{l}\text { At } 4 \text { weeks, } 8 \text { weeks, } 12 \text { weeks, } 4 \\
\text { months, } 6 \text { months, } 8 \text { months and } 10 \\
\text { months of age }\end{array}$ & $\begin{array}{l}\text { Focuses on expanding and extending sensitivity chain } \\
\text { practice, working through any challenges and flexibly } \\
\text { supporting parents and parental implementation of } \\
\text { sensitivity chain practice using ACT principles. Includes the } \\
\text { demonstration of a sensitivity chain whenever possible with } \\
\text { opportunities for reflection and feedback. }\end{array}$ \\
\hline
\end{tabular}

ACT, Acceptance and Commitment Therapy; ENACT, ENvironmental enrichment for infants; parenting with Acceptance and Commitment Therapy.

their own pace, with completion before their babies reach 8 weeks of age.

Mothers will be encouraged to engage in regular practice of sensitivity chains, with a goal dose of $30 \mathrm{~min}$ per day $/ 5$ days per week from 2 weeks of age and throughout the first year (total dose of approximately 125 hours). The social reciprocity intervention will be integrated into ordinary everyday interactions, including feeding, nappy changes and playful interactions. Consultation sessions will be conducted when the infant is 4 weeks, 8 weeks, 12 weeks, 4 months, 6 months, 8 months and 10 months of age, with capacity for additional sessions as needed. Consultations will support mothers in finding opportunities to practice within everyday life, in tailoring interactions to their babies and in adapting to their babies' developmental stage and skills. Mothers will be encouraged to initiate a sensitivity chain during the consultation, for the clinician's direct observation and feedback. In addition, clinical consultations will refer to ACT components, supporting maternal mental health throughout the first year. Clinical consultations will follow a specific protocol, and be recorded for fidelity (see table 1).

The primary target for recruitment and the intervention will be the mother, who will act as conduit to each infant's caregiving system. Other caregivers (eg, fathers and grandparents) will be given access to the ENACT edX course and will be welcome to participate in clinical consultations as applicable. Mothers will also be encouraged to teach all other significant caregivers the sensitivity chain intervention via direct demonstration.

\section{Fidelity}

The study clinician is experienced in working with families of children with neurodevelopmental disabilities, completed general training in ACT and also completed project-specific training via the ENACT intervention manual. The clinician will receive clinical supervision from KW to support fidelity. Course completion will be checked by the clinician. Clinical consultations will follow a specific protocol and will be recorded; $20 \%$ will be checked for fidelity (content and process) against the protocol. Qualitative feedback will be collected.

\section{Patient and public involvement}

Consumer feedback was sought on the protocol, the study forms and the intervention. Consumer feedback was positive, with some changes to the wordings made following the input.

\section{Study procedure}

Researchers will contact interested mothers to assess eligibility and provide detailed study information (see online supplementary file S1: study information sheet and consent form). Mothers will provide written consent prior to completing baseline assessments, and computergenerated block randomisation will then be used to randomise families (1:1) to intervention or CAU via Research Electronic Data Capture (REDCap). Families allocated to intervention will receive immediate access to ENACT. Families allocated to CAU will receive routine antenatal and postnatal care. 
Assessments will be conducted at baseline (prenatal), 3 months, 6 months and 12 months CA. Parents will complete questionnaire measures online; mother-infant relationship observations will be conducted via 20-minute video-recorded interactions and child development assessments will be undertaken at theUniversity of Queensland at 6 months and 12 months of age. While completing ENACT, parents will be invited to provide feedback and suggestions for course improvement.

\section{Measures}

Baseline assessments

The Parent Questionnaire collects (1) general demographic information (parent age, education, income and family composition) and (2) information relevant to the ASD context, such as parent's health history and details of the diagnosis of the first-degree relative (parent or sibling) with ASD. Further information regarding infant delivery, perinatal history, and feeding history will be collected postnatally by a brief phone interview.

The Broad Autism Phenotype Questionnaire (36 items) assesses ASD-like features in adults through self-report or an informant measure ${ }^{88}$ Participants rate how much each item applies to them on a 6-point Likert scale ${ }^{89}$ Internal consistency for the total scale is excellent $(\alpha=0.95)$ and there is good inter-item reliability. ${ }^{89}$

\section{Child assessments}

\section{Autism symptomatology}

The AOSI (12 months) will be the primary clinical outcome measure assessing intervention effect on infant development and severity of autism symptomatology at 12 months. ${ }^{62}$ It is an experimenter-led, semistructured observational assessment tool, developed for research purposes to study the emergence of ASD-related behavioural markers in infancy (6-18 months). ${ }^{61} 62$ Five standardised activities are delivered between two periods of free play, with a total of 18 items to be scored. Interrater reliability of total marker counts (number of items marked as atypical) and total scores, respectively, is good at 6 months $(0.68$ and 0.74$)$ and excellent at 12 months $(0.92$ and 0.93$) .{ }^{61}$ Test-retest reliability for total marker counts and total scores is fair to good at 0.68 and 0.61 , respectively. ${ }^{61}$ The AOSI differentiates between high-risk and low-risk infants at $12-14$ months $^{90-92}$.

The gap-overlap task (6 months and 12 months) ${ }^{63}$ is used to assess visual attention by measuring differences in the efficiency of orienting towards peripheral stimuli. A mix of social and non-social stimuli will be used. Two trial types will be contrasted: gap and overlap. In the gap condition, an interval of 200-250 ms separates the disappearance of the central stimulus and the appearance of the peripheral one (facilitation). In the overlap condition, the central stimulus remains visible and overlaps with the peripheral stimulus. This measures the ability to disengage from a central stimulus and to orient to a peripheral one. This difference between the gap and overlap times is called the gap effect. gap-overlap time, measured in ms, decreases from 6 months to 12 months for typically developing infants. ${ }^{159394}$ Infants who are later diagnosed with ASD consistently show an increase in gap-overlap time between 6 months and 12 months of age. ${ }^{159596}$ This has been called 'sticky attention'. Test-retest reliability of the gap-overlap task's gap effect is $r=0.50$ in infants of 10 months of age. ${ }^{63}$

\section{Neurodevelopmental and motor assessments}

The MSEL (6 months and 12 months) has been used in the cognitive assessment of infants and children from birth until 68 months of age..$^{30}{ }^{32-99}$ The MSEL has five scales: gross motor, VR, FM, GM, EL and RL, as well as an ELC score that is composed of the VR, FM, EL and RL subscales. The MSEL has demonstrated convergent and divergent construct validity in infants and children with ASD. ${ }^{100-102}$ Inter-rater reliability has been reported as high $(\mathrm{r}=0.91-0.99) .^{103}$

The GMA (3 months) is a predictive and discriminative tool that assesses infants' spontaneous motor activity from preterm to 20 weeks CA. ${ }^{104}$ Scoring is completed from a video recording with two full movement sequences required for pattern recognition (approximately $5 \mathrm{~min}$ ). ${ }^{104}$ During the fidgety period from 9 weeks to 20 weeks postterm, fidgety movements can be abnormal (exaggerated in amplitude and speed), sporadic (confined to a few body parts, never $>3 \mathrm{~s}$ between 9 and 16 weeks CA) or absent (fidgety movements not present between 9 and 16 weeks CA) (optimality scoring). ${ }^{104}$ Abnormal fidgety movements that are absent or abnormal at 12-14 weeks CA are highly predictive of cerebral palsy as well as other neurodevelopmental disabilities, including ASD. ${ }^{105} 106$ GMA will be scored by accredited blinded assessors. It will be used as a predictive tool, to better understand the sample.

The HINE (6 months and 12 months) is a standardised clinical neurodevelopmental assessment for infants from 2 months to 24 months of age. ${ }^{107}$ The HINE contains 26 items across 5 domains, summed to provide a global optimality score, and can differentiate between low-risk and high-risk late preterm and term newborns at 6 months and 12 months of age. ${ }^{108-110}$

The VABS-3 (12 months) is a standardised measure of adaptive behaviour, completed by caregivers and scored by a blinded assessor. ${ }^{69}$ Standard scores are generated for the four domains (communication, daily living skills, socialisation and motor skills) as well as a global score (Adaptive Behaviour Composite). It has good internal consistency, test-retest reliability, inter-interviewer reliability and validity for young children, including those with autism. ${ }^{69}$

\section{Infant regulation}

The BISQ (10 items; 3 months and 6 months) assesses parent-reported infant sleep patterns (nocturnal sleep duration, night waking and method of falling asleep), parent perception of infant sleep duration and sleeprelated (parent) behaviours for children from birth to 
36 months. It is well validated by comparisons with actigraphy, sleep diaries and caregiver-reported sleep. ${ }^{77} 78$

The CPQ (6 items; 3 months and 6 months) is a parent-report measure assessing: (1) the amount and time of day when infant crying occurs; (2) situations in which crying occurs; (3) whether the mother finds the crying distressing and seeks advice and help and (4) the mother's responses to crying. In comparison to 24-hour cry-fuss diaries kept by mothers, the $\mathrm{CPQ}$ showed moderate-to-good validity $(0.51-0.68)$ for total duration of crying scores. ${ }^{78}$

The ITSEA (165 items; 12 months) is a parent-report questionnaire used to assess social-emotional problems/ competencies in the domains of behavioural dysregulation and competence. The ITSEA has established concurrent validity, strong test-retest reliability $(\alpha=0.75-0.91)$ and good internal reliability for each subscale $(\alpha=0.86$ for dysregulation, $\alpha=0.87$ for externalising, $\alpha=0.85$ for internalising and $\alpha=0.89$ for competence). ${ }^{111} 112$ The ITSEA has been validated for 12 months CA and discriminates between low-risk and high-risk infants, particularly within the domain of dysregulation. ${ }^{76}$

The CSBS-DP (6 months and 12 months) evaluates the symbolic abilities and communication skills of children aged 6-24 months. ${ }^{73}$ It includes a 24-item Infant Toddler Checklist, which is used as a developmental screening tool to detect autism. ${ }^{113}$ The CSBS-DP has excellent internal consistency $(\alpha=0.86-0.92)$, good test-retest reliability and good construct and concurrent validity. ${ }^{73114}$

\section{Mother-infant relationship}

EAS (6 months): coders, blind to intervention condition, will use the EAS to score 20-minute naturalistic observations of parent-child interactions. ${ }^{67}$ The parent-child interaction will occur in the family's own home, with the parent instructed to interact with their child as they normally would. The observations will be recorded via the videoconferencing software Zoom. The EAS is used to measure quality of parent-child relationships across six scales: parental sensitivity, parental structuring, parental non-intrusiveness, parental non-hostility, child responsiveness and child involvement. ${ }^{67}$ The scales have high inter-rater reliability for the parent scales of sensitivity (0.95), structuring (0.87), non-intrusiveness $(0.81)$, nonhostility $(0.72)$ and the child scales of responsiveness (0.87) and involvement (0.87). ${ }^{115}$

The Emotional Availability-Self-Report (36 items; 3 months, 6 months and 12 months) is a parent-report questionnaire used to measure emotional availability in a dyadic relationship across five subscales: intrusiveness, hostility, mutual attunement, affect quality and capacity to involve the parent. Reliability ranges from 0.71 to 0.84 for all subscales except affect quality $(\alpha=0.49) .{ }^{115}$ All subscales (except for intrusiveness) have moderate correlations with the corresponding EAS observed subscales, thus supporting the validity of the self-report measure. ${ }^{115} 116$

\section{Maternal mental health}

The AAQ-II ( 7 items; baseline, 3 months, 6 months and 12 months) is a self-report questionnaire measuring psychological flexibility, the key target of ACT. ${ }^{66}$ The AAQ-II has good test-retest reliability, convergent validity and excellent internal consistency $(\alpha=0.94){ }^{66}$

The DASS-21 (baseline, 3 months, 6 months and 12 months) assesses symptoms of depression, anxiety and stress in adults. The DASS-21 produces three subscales, each with good internal consistency: the depression $(\alpha=0.91-0.97)$, anxiety $(\alpha=0.81-0.92)$ and stress $(\alpha=0.88-0.95)$ scales, ${ }^{117}$ and a total score. The DASS-21 has good convergent validity and acceptable discriminative validity. ${ }^{17}$

\section{Comparison group}

A comparison group of 30 healthy, low-risk infants will be recruited and assessed on the gap-overlap task and the HINE at 6 months and 12 months, and the AOSI at 12 months. This comparison data will support the interpretation of results, particularly for the novel gap-overlap task. To participate, the low-risk infant would need to have no first-degree relatives diagnosed with ASD, be born at term and have no other known developmental risk. The comparison group will be recruited through social media and word of mouth.

\section{Data collection and management}

The data will be entered onto the REDCap database in a potentially individually identifiable format. Once de-identified, the data will be stored in a re-identifiable format on a secure electronic database protected by the University of Queensland secure server, and only accessible to members of the research team.

\section{Statistical analysis}

Analysis (using STATA or SPSS 25.00) will follow standard methods for RCTs using comparisons between the two groups (eg, general linear models and Analysis of Covariance) and intention-to-treat analyses. Assumptions for parametric analyses will be assessed. Baseline scores will be included as covariates. Missing data will be handled using pro-rating and/or estimation maximisation depending on the assessment and pattern of missingness.

\section{Monitoring}

\section{Data monitoring}

As this is a trial of a very early intervention with low risk, a data monitoring committee is not required. Any adverse events, particularly negative developmental outcomes, will be recorded and reported to the ethics committees and in the published results.

\section{Harms}

This study should not pose risks beyond those of everyday living. Any participants experiencing undue psychological distress will be referred to their general practitioner. For infants scoring at high developmental risk on the 12 month assessments infants' general practitioners/paediatricians and parents will be notified. All families will be sent 
a paediatrician's report detailing 12-month developmental assessment results.

\section{ETHICS AND DISSEMINATION}

ENACT should support mothers' mental health and may also support infant development. Ethical approval has been obtained from the Children's Health Queensland Hospital and Health Service Human Research Ethics Committee (HREC/19/QCHQ/50131) and the University of Queensland Human Research Ethics Committee (2019000558). Study results will be disseminated through scientific journal publications and conference presentations. If shown to be effective, edX facilitates easy dissemination at minimal cost.

\section{DISCUSSION}

This study will test the efficacy of an innovative, very early intervention for infants at risk of ASD, integrating early social reciprocity intervention with parental mental health and parent-child relationship support. Potential limitations include recruitment and retention of parents with significant caregiving responsibilities; possible overestimation of anticipated effect size; substantial burden of assessment for mothers; use of parent-report measures of infant regulation and limited ability to assess day-to-day intervention implementation by mothers in the home environment.

\section{Author affiliations}

${ }^{1}$ Queensland Cerebral Palsy and Rehabilitation Research Centre, Faculty of Medicine, UQ Child Health Research Centre, The University of Queensland, Brisbane, Queensland, Australia

${ }^{2}$ Parenting and Family Support Centre, School of Psychology, The University of Queensland, Brisbane, Queensland, Australia

${ }^{3} U Q$ Child Health Research Centre, Faculty of Medicine, The University of Queensland, Brisbane, Queensland, Australia

${ }^{4}$ Centre for Clinical Trials in Rare Neurodevelopmental Disorders, Child Development Program, Child Youth and Community Health Services, Children's Health Queensland, Brisbane, Queensland, Australia

Correction notice This article has been corrected since it was published. The affiliations have been updated.

Acknowledgements This work was supported by two University of Queensland Graduate School Scholarships (AM and KK), a Children's Hospital Foundation Early Career Fellowship (AEM; award ref. no. 50223), an NHMRC Research Fellowship (RNB; 1105038) and a philanthropic donation.

Contributors All authors contributed to the conception and design of this study, contributed to drafting and critical revision of the manuscript and approved the final version of the manuscript to be submitted for publication. KW and AMG designed the ENACT intervention. KK, KW and AEM contributed to the design in terms of parental mental health and parent-child relationship assessment. AMG, $\mathrm{KW}, \mathrm{HH}$ and RNB contributed to the design in terms of motor, cognitive and autistic symptomatology assessment.

Funding This work is supported by two University of Queensland Graduate School Scholarships (UQGSS) (AM and KK), a Children's Hospital Foundation Early Career Fellowship (AEM; award ref. no. 50223), an NHMRC Research Fellowship (RNB; 1105038) and a private philanthropic donation. The private philanthropic donor does not have any role in this study

Competing interests ENACT was developed from PACT, an intervention developed by researchers at The University of Queensland, including KW and RNB. AMG and KW developed the very early intervention component of edX. ENACT has been developed using the online platform edX.
Patient and public involvement Patients and/or the public were involved in the design, or conduct, or reporting, or dissemination plans of this research. Refer to the Methods section for further details.

Patient consent for publication Not required.

Provenance and peer review Not commissioned; externally peer reviewed.

Open access This is an open access article distributed in accordance with the Creative Commons Attribution Non Commercial (CC BY-NC 4.0) license, which permits others to distribute, remix, adapt, build upon this work non-commercially, and license their derivative works on different terms, provided the original work is properly cited, appropriate credit is given, any changes made indicated, and the use is non-commercial. See: http://creativecommons.org/licenses/by-nc/4.0/.

ORCID iD

Koa Whittingham http://orcid.org/0000-0002-5344-9907

\section{REFERENCES}

1 American Psychiatric Association. Diagnostic and statistical manual of mental disorders: DSM-5. 5th ed. Washington: American Psychiatric Publishing, 2013.

2 Mandy WP, Charman T, Skuse DH. Testing the construct validity of proposed criteria for DSM-5 autism spectrum disorder. J Am Acad Child Adolesc Psychiatry 2012;51:41-50.

3 Chaste P, Leboyer M. Autism risk factors: genes, environment, and gene-environment interactions. Dialogues Clin Neurosci 2012;14:281-92.

4 Veenstra-VanderWeele J, Wender CLA. Challenge and potential for research on gene-environment interactions in autism spectrum disorder. In: Tolan PH, Leventhal BL, eds. Gene-Environment transactions in developmental psychopathology. New York: Springer, 2017.

5 Australian Institute of Health and Welfare. Autism in Australia, 2017. Available: https://www.aihw.gov.au/reports/disability/autism-inaustralia/contents/autism

6 Kogan MD, Vladutiu CJ, Schieve LA, et al. The prevalence of parent-reported autism spectrum disorder among US children. Pediatrics 2018;142.

7 Baio J, Wiggins L, Christensen DL, et al. Prevalence of autism spectrum disorder among children aged 8 years - autism and developmental disabilities monitoring network, 11 sites, United States, 2014. MMWR Surveill Summ 2018;67:1-23.

8 Bent CA, Dissanayake C, Barbaro J. Mapping the diagnosis of autism spectrum disorders in children aged under 7 years in Australia, 2010-2012. Med J Aust 2015;202:317-20.

9 Ozonoff S, Young GS, Carter A, et al. Recurrence risk for autism spectrum disorders: a baby siblings research consortium study. Pediatrics 2011;128:e488-95.

10 Messinger DS, Young GS, Webb SJ, et al. Early sex differences are not autism-specific: a baby siblings research consortium (BSRC) study. Mol Autism 2015;6:32.

11 Charman T, Young GS, Brian J, et al. Non-ASD outcomes at 36 months in siblings at familial risk for autism spectrum disorder (ASD): a baby siblings research consortium (BSRC) study. Autism Res 2017;10:169-78.

12 Shephard E, Milosavljevic B, Pasco G, et al. Mid-childhood outcomes of infant siblings at familial high-risk of autism spectrum disorder. Autism Res 2017;10:546-57.

13 Miller M, losif A-M, Young GS, et al. School-age outcomes of infants at risk for autism spectrum disorder. Autism Res 2016;9:632-42.

14 Bedford R, Gliga T, Shephard E, et al. Neurocognitive and observational markers: prediction of autism spectrum disorder from infancy to mid-childhood. Mol Autism 2017;8:49.

15 Clifford SM, Hudry K, Elsabbagh M, et al. Temperament in the first 2 years of life in infants at high-risk for autism spectrum disorders. $J$ Autism Dev Disord 2013;43:673-86.

16 Leonard HC, Bedford R, Charman T, et al. Motor development in children at risk of autism: a follow-up study of infant siblings. Autism 2014;18:281-91.

17 Sacrey L-AR, Armstrong VL, Bryson SE, et al. Impairments to visual disengagement in autism spectrum disorder: a review of experimental studies from infancy to adulthood. Neurosci Biobehav Rev 2014;47:559-77.

18 Franchini M, Duku E, Armstrong V, et al. Variability in verbal and nonverbal communication in infants at risk for autism spectrum disorder: predictors and outcomes. J Autism Dev Disord 2018;48:3417-31. 
19 Jones EJH, Gliga T, Bedford R, et al. Developmental pathways to autism: a review of prospective studies of infants at risk. Neurosci Biobehav Rev 2014;39:1-33.

20 Johnson $\mathrm{MH}$, Gliga T, Jones $\mathrm{E}$, et al. Annual research review: infant development, autism, and ADHD-early pathways to emerging disorders. J Child Psychol Psychiatry 2015;56:228-47.

21 Charman T. Mapping early symptom trajectories in autism spectrum disorder: lessons and challenges for clinical practice and science. $J$ Am Acad Child Adolesc Psychiatry 2018;57:820-1.

22 Elsabbagh $\mathrm{M}$, Johnson $\mathrm{MH}$. Autism and the social brain: the firstyear puzzle. Biol Psychiatry 2016;80:94-9.

23 Holmboe K, Elsabbagh M, Volein A, et al. Frontal cortex functioning in the infant broader autism phenotype. Infant Behav Dev 2010;33:482-91.

24 Estes A, Zwaigenbaum L, Gu H, et al. Behavioral, cognitive, and adaptive development in infants with autism spectrum disorder in the first 2 years of life. J Neurodev Disord 2015;7:24.

25 Trevarthen C, Delafield-Butt JT. Autism as a developmental disorder in intentional movement and affective engagement. Front Integr Neurosci 2013;7.

26 Tsang T. Mechanisms conferring risk versus resilience for autism spectrum disorder in early infancy. UCLA 2018

27 Conti E, Calderoni S, Marchi V, Sara E, Viviana E, et al. The first 1000 days of the autistic brain: a systematic review of diffusion imaging studies. Front Hum Neurosci 2015;9:159.

28 Wolff JJ, Jacob S, Elison JT. The journey to autism: insights from neuroimaging studies of infants and toddlers. Dev Psychopathol 2018;30:479-95.

29 Emerson RW, Adams C, Nishino T, et al. Functional neuroimaging of high-risk 6-month-old infants predicts a diagnosis of autism at 24 months of age. Sci Transl Med 2017;9. doi:10.1126/scitranslmed. aag2882

30 Green J, Charman T, Pickles A, et al. Parent-mediated intervention versus no intervention for infants at high risk of autism: a parallel, single-blind, randomised trial. Lancet Psychiatry 2015;2:133-40.

31 Baranek GT, Watson LR, Turner-Brown L, et al. Preliminary efficacy of adapted responsive teaching for infants at risk of autism spectrum disorder in a community sample. Autism Res Treat 2015;2015:386951

32 Watson LR, Crais ER, Baranek GT, et al. Parent-Mediated intervention for one-year-olds screened as at-risk for autism spectrum disorder: a randomized controlled trial. J Autism Dev Disord 2017;47:3520-40.

33 Whitehouse AJO, Varcin KJ, Alvares GA, et al. Pre-emptive intervention versus treatment as usual for infants showing early behavioural risk signs of autism spectrum disorder: a singleblind, randomised controlled trial. Lancet Child Adolesc Health 2019;3:605-15.

34 Green J, Pickles A, Pasco G, et al. Randomised trial of a parent-mediated intervention for infants at high risk for autism: longitudinal outcomes to age 3 years. J Child Psychol Psychiatry 2017;58:1330-40.

35 Kasari C, Siller M, Huynh LN, et al. Randomized controlled trial of parental responsiveness intervention for toddlers at high risk for autism. Infant Behav Dev 2014;37:711-21.

36 Jones EJH, Dawson G, Kelly J, et al. Parent-delivered early intervention in infants at risk for ASD: effects on electrophysiological and habituation measures of social attention. Autism Res 2017;10:961-72.

37 Hartford D, Knotek S, Mesibov G, et al. A responsive teaching intervention for parents of children identified as at risk for an autism spectrum disorder at 12 months [ProQuest Dissertations Publishing], 2010. Available: http://search.proquest.com/docview/ 815242599/

38 Carter AS, Messinger DS, Stone WL, et al. A randomized controlled trial of Hanen's 'More Than Words' in toddlers with early autism symptoms. J Child Psychol Psychiatry 2011;52:741-52.

39 Rogers SJ, Estes A, Lord C, et al. Effects of a Brief Early Start Denver Model (ESDM)-Based Parent Intervention on Toddlers at Risk for Autism Spectrum Disorders: A Randomized Controlled Trial. Journal of the American Academy of Child \& Adolescent Psychiatry 2012;51:1052-65.

40 Field T. Infants of depressed mothers. Dev Psychopathol 1992;4:49-66.

41 Quintero N, Mclntyre LL, Adjustment S. Sibling adjustment and maternal well-being: an examination of families with and without a child with an autism spectrum disorder. Focus Autism Other Dev Disabl 2010;25:37-46.

42 Yorke I, White P, Weston A, et al. The association between emotional and behavioral problems in children with autism spectrum disorder and psychological distress in their parents: a systematic review and meta-analysis. J Autism Dev Disord 2018;48:3393-415.

43 Nicholas DB, Zwaigenbaum L, Ing S, et al. "Live it to understand it": the experiences of mothers of children with autism spectrum disorder. Qual Health Res 2016;26:921-34.

44 Wiggins LD, Rubenstein E, Daniels J, et al. A phenotype of childhood autism is associated with preexisting maternal anxiety and depression. J Abnorm Child Psychol 2018.

45 Yirmiya N, Shaked M. Psychiatric disorders in parents of children with autism: a meta-analysis. J Child Psychol Psychiatry 2005:46:69-83.

46 Goodman SH, Rouse MH, Connell AM, et al. Maternal depression and child psychopathology: a meta-analytic review. Clin Child Fam Psychol Rev 2011:14:1-27.

47 Seidman I, Yirmiya N, Milshtein S, et al. The broad autism phenotype questionnaire: mothers versus fathers of children with an autism spectrum disorder. J Autism Dev Disord 2012;42:837-46

48 Rubenstein E, Chawla D. Broader autism phenotype in parents of children with autism: a systematic review of percentage estimates. J Child Fam Stud 2018;27:1705-20.

49 Rubenstein E, Wiggins LD, Schieve LA, et al. Associations between parental broader autism phenotype and child autism spectrum disorder phenotype in the study to explore early development. Autism 2019;23:436-48.

50 Ingersoll B, Hambrick DZ. The relationship between the broader autism phenotype, child severity, and stress and depression in parents of children with autism spectrum disorders. Res Autism Spectr Disord 2011;5:337-44.

51 Whittingham K, Sheffield J, Boyd RN. Parenting acceptance and commitment therapy: a randomised controlled trial of an innovative online course for families of children with cerebral palsy. BMJ Open 2016;6:e012807.

52 Lowe JR, MacLean PC, Duncan AF, et al. Association of maternal interaction with emotional regulation in 4- and 9-month infants during the still face paradigm. Infant Behav Dev 2012;35:295-302.

53 Harker CM, Ibañez LV, Nguyen TP, et al. The effect of parenting style on social Smiling in infants at high and low risk for ASD. J Autism Dev Disord 2016;46:2399-407.

54 Sullivan K, Stone WL, Dawson G. Potential neural mechanisms underlying the effectiveness of early intervention for children with autism spectrum disorder. Res Dev Disabil 2014;35:2921-32.

55 LeBlanc JJ, Fagiolini M. Autism: a "critical period" disorder? Neural Plast 2011;2011:1-17.

56 Killmeyer S, Kaczmarek L. Parent training and joint engagement in young children with autism spectrum disorder. Autism Dev Lang Impair 2017;2

57 Elsabbagh M, Gliga T, Pickles A, et al. The development of face orienting mechanisms in infants at-risk for autism. Behav Brain Res 2013;251:147-54

58 Elsabbagh M, Holmboe K, Gliga T, et al. Social and attention factors during infancy and the later emergence of autism characteristics. Prog Brain Res 2011;189:195-207.

59 Wan MW, Green J, Elsabbagh M, et al. Parent-Infant interaction in infant siblings at risk of autism. Res Dev Disabil 2012;33:924-32.

60 Wan MW, Green J, Elsabbagh M, et al. Quality of interaction between at-risk infants and caregiver at 12-15 months is associated with 3-year autism outcome. J Child Psychol Psychiatry 2013:54:763-71.

61 Bryson SE, Zwaigenbaum L, McDermott C, et al. The autism observation scale for infants: scale development and reliability data. J Autism Dev Disord 2008;38:731-8.

62 Bryson S, Zwaigenbaum L. Autism observation scale for infants. In: Patel V, Preedy V, Martin C, eds. Comprehensive guide to autism. New York: Springer, 2014: 299-310.

63 Cousijn J, Hessels RS, Van der Stigchel S, et al. Evaluation of the psychometric properties of the gap-overlap task in 10-month-old infants. Infancy 2017;22:571-9.

64 Elsabbagh M, Volein A, Holmboe K, et al. Visual orienting in the early broader autism phenotype: disengagement and facilitation. $J$ Child Psychol Psychiatry 2009;50:637-42.

65 Brown TA, Chorpita BF, Korotitsch W, et al. Psychometric properties of the depression anxiety stress scales (DASS) in clinical samples. Behav Res Ther 1997;35:79-89.

66 Mitchell AE, Whittingham K, Steindl S, et al. Feasibility and acceptability of a brief online self-compassion intervention for mothers of infants. Arch Womens Ment Health 2018;21:553-61.

67 Biringen Z, Derscheid D, Vliegen N, et al. Emotional availability (EA): theoretical background, empirical research using the EA scales, and clinical applications. Develop Rev 2014;34:114-67. 
68 Burns TG, King TZ, Spencer KS. Mullen scales of early learning: the utility in assessing children diagnosed with autism spectrum disorders, cerebral palsy, and epilepsy. Appl Neuropsychol Child 2012;2:1-10.

69 Sparrow SS, Cicchetti DV, Saulnier C. Vineland adaptive behavior scales, (Vineland-3). 3rd ed. Bloomington, MN: Pearson, 2016.

70 Haataja L, Cowan F, Mercuri E, et al. Application of a scorable neurologic examination in healthy term infants aged 3 to 8 months. J Pediatr 2003;143:546-46.

71 Haataja L, Mercuri E, Regev R, et al. Optimality score for the neurologic examination of the infant at 12 and 18 months of age. $J$ Pediatr 1999;135:153-61.

72 Morgan C, Honan I, Allsop A, et al. Psychometric properties of assessments of cognition in infants with cerebral palsy or motor impairment: a systematic review. J Pediatr Psychol 2018.

73 Wetherby AM, Allen L, Cleary J, et al. Validity and reliability of the communication and symbolic behavior scales developmental profile with very young children. J Speech Lang Hear Res 2002;45:1202-18

74 Luyster RJ, Kadlec MB, Carter A, et al. Language assessment and development in toddlers with autism spectrum disorders. J Autism Dev Disord 2008;38:1426-38.

75 Watt N, Wetherby A, Shumway S. Prelinguistic predictors of language outcome at 3 years of age. J Speech Lang Hear Res 2006;49:1224-37

76 Sanner N, Smith L, Wentzel-Larsen T, et al. Early identification of social-emotional problems: applicability of the Infant-Toddler social emotional assessment (ITSEA) at its lower age limit. Infant Behav Dev 2016;42:69-85.

77 Teng A, Bartle A, Sadeh A, et al. Infant and toddler sleep in Australia and New Zealand. J Paediatr Child Health 2012;48:268-73.

78 Wolke D, Meyer R, Gray P. Validity of the crying pattern questionnaire in a sample of excessively crying babies. $J$ Reprod Infant Psychol 1994;12:105-14.

79 Jones EJH, Venema K, Earl R, et al. Reduced engagement with social stimuli in 6-Month-Old infants with later autism spectrum disorder: a longitudinal prospective study of infants at high familial risk. J Neurodev Disord 2016;8:7.

80 Whittingham K, Sanders M, McKinlay L, et al. Interventions to reduce behavioral problems in children with cerebral palsy: an RCT. Pediatrics 2014;133:1249-57.

81 Whittingham K, Sanders MR, McKinlay L, et al. Parenting intervention combined with acceptance and commitment therapy: a trial with families of children with cerebral palsy. J Pediatr Psycho 2016;41:531-42.

82 Brown FL, Whittingham K, Boyd RN, et al. Improving child and parenting outcomes following paediatric acquired brain injury: a randomised controlled trial of stepping stones triple $P$ plus acceptance and commitment therapy. J Child Psychol Psychiatry 2014:55:1172-83.

83 Brown FL, Whittingham K, McKinlay L, et al. Efficacy of stepping stones triple $P$ plus a stress management adjunct for parents of children with an acquired brain injury: the protocol of a randomised controlled trial. Brain Impairment 2013;14:253-69.

84 Whittingham K, Coyne LW. Acceptance and commitment therapy : the clinician's guide for supporting parents. London: Academic Press, 2019.

85 Whittingham K, Douglas P. Optimizing parent-infant sleep from birth to 6 months: a new paradigm. Infant Ment Health J 2014;35:614-23.

86 Zeifman DMSt James-Roberts I. Parenting the crying infant. Curr Opin Psychol 2017;15:149-54.

87 Whittingham K. Parenting in context. J Contextual Behav Sci 2014;3:212-5.

88 Hurley RS, Losh M, Parlier M, et al. The broad autism phenotype questionnaire. J Autism Dev Disord 2007;37:1679-90.

89 Sasson NJ, Lam KSL, Childress D, et al. The broad autism phenotype questionnaire: prevalence and diagnostic classification. Autism Res 2013;6:134-43.

90 Brian J, Bryson SE, Garon N, et al. Clinical assessment of autism in high-risk 18-month-olds. Autism 2008;12:433-56.

91 Gammer I, Bedford R, Elsabbagh M, et al. Behavioural markers for autism in infancy: scores on the autism observational scale for infants in a prospective study of at-risk siblings. Infant Behav Dev 2015;38:107-15.

92 Georgiades S, Szatmari P, Zwaigenbaum L, et al. A prospective study of autistic-like traits in unaffected siblings of probands with autism spectrum disorder. JAMA Psychiatry 2013;70:42-8.

93 Matsuzawa M, Shimojo S. Infants' fast saccades in the gap paradigm and development of visual attention. Infant Behav Develop 1997;20:449-55.
94 McConnell BA, Bryson S. Visual attention and temperament: developmental data from the first 6 months of life. Infant Behav Develop 2005;28:537-44.

95 Zwaigenbaum L, Bryson S, Rogers T, et al. Behavioral manifestations of autism in the first year of life. Int $J$ Dev Neurosci 2005;23:143-52.

96 Bryson S, Garon N, McMullen T, et al. Impaired disengagement of attention and its relationship to emotional distress in infants at high-risk for autism spectrum disorder. J Clin Exp Neuropsychol 2018;40:487-501.

97 Brian AJ, Roncadin C, Duku E, et al. Emerging cognitive profiles in high-risk infants with and without autism spectrum disorder. Res Autism Spectr Disord 2014;8:1557-66.

98 Garon N, Zwaigenbaum L, Bryson S, et al. Temperament and its association with autism symptoms in a high-risk population. $J$ Abnor Child Psychol 2016;44:757-69.

99 Ozonoff S, Young GS, Brian J, et al. Diagnosis of autism spectrum disorder after age 5 in children evaluated longitudinally since infancy. J Am Acad Child Adolesc Psychiatry 2018;57:849-57.

100 Bishop SL, Guthrie W, Coffing M, et al. Convergent validity of the Mullen scales of early learning and the differential ability scales in children with autism spectrum disorders. Am J Intellect Dev Disabil 2011;116:331-43.

101 Swineford LB, Guthrie W, Thurm A. Convergent and divergent validity of the Mullen scales of early learning in young children with and without autism spectrum disorder. Psychol Assess 2015;27:1364-78.

102 Akshoomoff N. Use of the Mullen scales of early learning for the assessment of young children with autism spectrum disorders. Child Neuropsychol 2006;12:269-77.

103 Mullen EM. Mullen scales of early learning. Circle Pines: American Guidance Service, 1995

104 Einspieler C, Prechtl HFR, Arend B, et al. Prechtl's method on the qualitative assessment of general movements in pretermterm and young infants. Clin Dev Med 2004;167:1-91.

105 Einspieler C, Sigafoos J, Bartl-Pokorny KD, Bolte S, et al. Highlighting the first 5 months of life: general movements in infants later diagnosed with autism spectrum disorder or Rett syndrome. Res Autism Spectr Disord 2014;8:286-91.

106 Zappella M, Einspieler C, Bartl-Pokorny KD, et al. What do home videos tell us about early motor and socio-communicative behaviours in children with autistic features during the second year of life â€" an exploratory study. Early Hum Dev 2015;91:569-75.

107 Haataja L, Mercuri E, Regev R, et al. Optimality score for the neurologic examination of the infant at 12 and 18 months of age. $J$ Pediatr 1999;135:153-61.

108 Maitre NL, Chorna O, Romeo DM, et al. Implementation of the Hammersmith infant neurological examination in a high-risk infant follow-up program. Pediatr Neurol 2016;65:31-8.

109 Romeo DM, Brogna C, Sini F, et al. Early psychomotor development of low-risk preterm infants: influence of gestational age and gender. Eur J Paediatr Neurol 2016;20:518-23.

110 Chatziioannidis I, Kyriakidou M, Exadaktylou S, et al. Neurological outcome at 6 and 12 months corrected age in hospitalised late preterm infants -A prospective study. Eur J Paediatr Neurol 2018;22:602-9.

111 Briggs-Gowan M, Carter A. Infant toddler social \& emotional assessment (ITSEA) manual. Massachusetts: PsychCorp, 2001.

112 Carter A, Briggs-Gowan M. ITSEA: infant-toddler social and emotional assessment. Massachusetts: PsychCorp, 2006.

113 Wetherby AM, Woods J, Allen L, et al. Early indicators of autism spectrum disorders in the second year of life. J Autism Dev Disord 2004:34:473-93.

114 Eadie PA, Ukoumunne O, Skeat J, et al. Assessing early communication behaviours: structure and validity of the communication and symbolic behaviour Scales-Developmental profile (CSBS-DP) in 12-month-old infants. Int J Lang Commun Disord 2010;45:572-85.

115 Vliegen N, Luyten P, Biringen Z. A Multimethod perspective on emotional availability in the postpartum period. Parenting 2009;9:228-43.

116 Luyten P, Mayes LC, Nijssens L, et al. The parental reflective functioning questionnaire: development and preliminary validation. PLoS One 2017;12:e0176218.

117 Gloster AT, Rhoades HM, Novy D, et al. Psychometric properties of the depression anxiety and stress scale-21 in older primary care patients. J Affect Disord 2008;110:248-59. 